J. Igusa

Nagoya Math. J.

Vol. 53 (1974), 211-233

\title{
ON A CERTAIN POISSON FORMULA ${ }^{1}$
}

\author{
JUN-ICHI IGUSA
}

Introduction. Let $G$ denote a locally compact commutative group with a lattice $\Gamma, G^{*}$ its dual, and $\left\langle g, g^{*}\right\rangle=g^{*}(g)$ for every $\left(g, g^{*}\right)$ in $G \times G^{*}$; let $\Gamma_{*}$ denote the annihilator of $\Gamma$ in $G^{*}$ and $d g$ the Haar measure on $G$ such that $G / \Gamma$ is of measure 1. Finally, let $F$ denote an $L^{1}$-function on $G$ and $F^{*}$ its Fourier transform defined by

$$
F^{*}\left(g^{*}\right)=\int_{G} F(g)\left\langle g, g^{*}\right\rangle d g \text {. }
$$

Then, under suitable conditions on $F$, we have

$$
\sum_{\gamma \in \Gamma} F(\gamma)=\sum_{\gamma^{*} \in \Gamma_{*}} F^{*}\left(\gamma^{*}\right)
$$

in which both sides are absolutely convergent. This is a classical Poisson formula.

Let $X$ denote a locally compact commutative group, $d x$ a Haar measure on $X$, and $f$ a continuous mapping of $X$ to the above group $G$; for every $\Phi$ in the Schwartz-Bruhat space $S(X)$ of $X$, define a function $F^{*}=F_{\Phi}^{*}$ on $G^{*}$ as

$$
F_{\phi}^{*}\left(g^{*}\right)=\int_{X} \Phi(x)\left\langle f(x), g^{*}\right\rangle d x
$$

Then, under suitable conditions on $f$, the Haar measure $d x$ decomposes into a family of tempered measures $d \mu_{g}$, where $\operatorname{Supp}\left(d \mu_{g}\right)$ is contained in $f^{-1}(g)$ for every $g$ in $G$, such that the above Poisson formula holds for $F=F_{\Phi}$ defined by

$$
F_{\emptyset}(g)=\int_{X} \Phi(x) d \mu_{g}(x)
$$

This variant is due to Weil [9]; it is an "abstract form" of the Siegel formula.

Received October 12, 1973.

1 This work was partially supported by the National Science Foundation. 
Let $k$ denote a global field, i.e., a number field or a function field of one variable with a finite constant field; let the subscripts $A$ and $k$ denote the adelization relative to $k$ and the taking of $k$-rational points, respectively; let $\psi$ denote a non-trivial character of $k_{A} / k$ and identify $k_{A}$ with its dual by $\left(i, i^{*}\right) \rightarrow \psi\left(i i^{*}\right)$. We shall fix a universal domain $K$ containing $k$ and identify $K$ with an affine line over the prime field. We shall change our notation slightly: let $X$ denote an affine space and $f$ a morphism of $X$ to $K$ defined over $k$; let $|d x|_{A}$ denote the Haar measure on $X_{A}$ such that $X_{A} / X_{k}$ is of measure 1 . Then we can take $k_{A}$ as $G=G^{*}, k$ as $\Gamma=\Gamma_{*}, X_{A}$ as $X,|d x|_{A}$ as $d x$, and $f_{A}$ as $f$. During the past several years, we became interested in proving a Poisson formula (of Weil's type) in the above setup. In this paper, we shall consider the special case where $f$ is homogeneous of degree at least 2 and "strongly non-degenerate" in the sense that it is submersive everywhere except at the origin 0 of $X$. We shall show, in that case, that the Poisson formula holds if char $(k)$ does not divide $\operatorname{deg}(f)$ and

$$
\operatorname{dim}(X)>2 \operatorname{deg}(f)
$$

In this formula, everything is explicitly defined; for a complete statement, we refer to $\S 5$, Th. 5 . It appears that the simplicity of the above condition is quite remarkable. We have included an additional section on some numerical coefficients of certain asymptotic expansions.

1. A review of some results. We shall keep the notation in the introduction. Let $v$ denote a valuation on the global field $k$ and $k_{v}$ the corresponding local field; let $\psi_{v}$ denote the product of the canonical injection $k_{v} \rightarrow k_{A}$ and the non-trivial character $\psi$ of $k_{A} / k$. We recall that $X$ is an affine space defined over $k$; we introduce coordinates in $X$ with respect to a $k$-base of $X_{k}$. Let $\left(x_{1}, \cdots, x_{n}\right),\left(y_{1}, \cdots, y_{n}\right)$ denote coordinates of $x, y$ in $X$; then $[x, y]=x_{1} y_{1}+\cdots+x_{n} y_{n}$ defines a non-degenerate symmetric bilinear form on $X \times X$. Let $X_{v}$ denote the vector space over $k_{v}$ of $k_{v}$-rational points of $X$; let $|d x|_{v}$ denote the autodual (or "self-dual") measure on $X_{v}$ relative to the bicharacter $(x, y) \rightarrow \psi_{v}([x, y])$ of $X_{v} \times X_{v}$. Then the restricted product measure of all $|d x|_{v}$ becomes the autodual measure on $X_{A}$ relative to the bicharacter $(x, y) \rightarrow \psi([x, y])$ of $X_{A} \times X_{A}$; this measure coincides with the Haar measure $|d x|_{A}$ on $X_{A}$ such that $X_{A} / X_{k}$ is of measure 1 . 
If we take 1 as a $k$-base of $k$, what we have said can be applied to the universal domain $K$ instead of $X$ : we shall denote by $|d i|_{v}$ the autodual measure on $k_{v}$ relative to the bicharacter $\left(i, i^{*}\right) \rightarrow \psi_{v}\left(i i^{*}\right)$ of $k_{v}$ $\times k_{v}$ and by $|d i|_{A}$ the restricted product measure of all $|d i|_{v}$, etc. We shall denote by ||$_{v}$ the absolute value on $k_{v}$ defined by $\left|d\left(i_{0} i\right)\right|_{v}=\left|i_{0}\right|_{v}|d i|_{v}$ for every $i_{0} \neq 0$ in $k_{v}$.

We recall that $f: X \rightarrow K$ is a morphism defined over $k$; it gives rise to a continuous mapping, in fact a $k_{v}$-analytic mapping, $f_{v}: X_{v} \rightarrow k_{v}$ for every $v$. If there is no ambiguity, we shall denote $f_{v}$ also by $f$. Let $X^{\prime}$ denote the set of points of $X$ where $f$ is submersive, i.e., where the cotangent vector $d f$ does not vanish; then $X^{\prime}$ is a Zariski open subset of $X$ defined over $k$. We observe that $f$ is strongly non-degenerate if and only if $X-X^{\prime} \subset\{0\}$; for a moment, we shall only assume that $X-X^{\prime} \subset f^{-1}(0)$. We put $U(i)=f^{-1}(i) \cap X^{\prime}$ for every $i$ in $K$; we have $U(i)=f^{-1}(i)$ if $i \neq 0$. For every $i$ in $k_{v}$, let $U(i)_{v}$ denote the set of $k_{v^{-}}$ rational points of $U(i)$; then $U(i)_{v}$ becomes a $k_{v}$-analytic manifold, and the union of all $U(i)_{v}$ coincides with the similarly defined open subset $X_{v}^{\prime}$ of $X_{v}$. Moreover, there exists a Borel measure $\left|\theta_{i}\right|_{v}$ on each $U(i)_{v}$ such that

$$
\int_{X_{v}} \phi(x)|d x|_{v}=\int_{k_{v}}\left(\int_{U(i)_{v}} \phi(x)\left|\theta_{i}(x)\right|_{v}\right)|d i|_{v}
$$

for every continuous function $\phi$ on $X_{v}$ with compact support contained in $X_{v}^{\prime}$; the measure $\left|\theta_{i}\right|_{v}$ admits an explicit analytic expression; cf. [9], pp. 12-13.

We define a function $F_{\Phi}$ on $k_{v}^{\times}=k_{v}-\{0\}$ for every $\Phi$ in the SchwartzBruhat space $\mathscr{S}\left(X_{v}\right)$ of $X_{v}$ as

$$
F_{\Phi}(i)=\int_{U(i)_{v}} \Phi(x)\left|\theta_{i}(x)\right|_{v} .
$$

We also define a function $F_{\phi}^{*}$ on $k_{v}$ as

$$
F_{\phi}^{*}\left(i^{*}\right)=\int_{X_{v}} \Phi(x) \psi_{v}\left(f_{v}(x) i^{*}\right)|d x|_{v}
$$

Finally, for every quasicharacter $\omega$ of $k_{v}^{\times}$which is bounded around 0 , we put

$$
Z(\omega, \Phi)=\int_{X_{v}} \omega\left(f_{v}(x)\right) \Phi(x)|d x|_{v}
$$


In $\mathrm{AE}$ we developed a coherent theory of the above three types of functions; in the following, we shall recall some of our results:

Suppose first that $k_{v}$ is an $\boldsymbol{R}$-field, i.e., $v$ is archimedean; then, for every quasicharacter $\omega$ of $k_{v}^{\times}$and $t$ in $k_{v}^{\times}$, we have

$$
\omega(t)=|t|_{v}^{s}\left(|t|^{-1} t\right)^{p}
$$

in which $s$ is in $C$ and $p$ in $Z$; we have $p=0,1$ if $k_{v}=R$. Conversely, for every $s$ in $C$ and $p$ in $Z$, the above prescription defines a quasicharacter of $k_{v}^{\times}$. The complex power (or the "local zeta function") $Z(\omega, \Phi)$ has a meromorphic continuation to the whole complex Lie group of quasicharacters of $k_{v}^{\times}$with poles only on the negative real axis of the $s$-plane. If $-\lambda$ is a pole of $Z(\omega, \Phi)$ and

$$
\sum_{i=1}^{m \lambda} b_{\lambda, i}(p)(s+\lambda)^{-i}
$$

the principal part of its Laurent expansion around $-\lambda$, we have the following asymptotic expansion:

$$
F_{\omega}^{*}\left(\gamma^{-1} t\right) \approx \sum_{i} \sum_{i=1}^{m_{\lambda}} a_{\lambda, i}^{*}\left(|t|^{-1} t\right)|t|_{v}^{-\lambda}\left(\log |t|_{v}\right)^{i-1}
$$

as $|t|_{v} \rightarrow \infty$. The constant $\gamma$ on the left hand side is an element of $k_{v}^{\times}$ defined by $\psi_{v}(t)=\boldsymbol{e}(\gamma t), \boldsymbol{e}(2 \operatorname{Re}(\gamma t))$ for every $t$ in $k_{v}=\boldsymbol{R}, \boldsymbol{C}$, respectively. The coefficients $a_{\lambda, i}^{*}(u)$ on the right hand side are determined by $b_{\lambda, i}(p)$ as follows: put $m_{v}=2,2 \pi, d=\frac{1}{2}, 1$ for $k_{v}=R, C$, respectively, and

$$
b_{p}(s)=i^{|p|}(2 d \pi)^{d(1-2 s)} \Gamma\left(d s+\frac{1}{2}|p|\right) / \Gamma\left(d(1-s)+\frac{1}{2}|p|\right) ;
$$

then

$$
\begin{gathered}
a_{\lambda, i}^{*}(u)=\left(1 / m_{v}\right) \sum_{p}\left(\sum_{j=1}^{m_{\lambda}}\left((-1)^{i+j} /(i-1) !(j-i) !\right) b_{\lambda, j}(p)\right. \\
\left.\cdot\left(d^{j-i} b_{p}(s) / d s^{j-i}\right)_{s=\lambda}\right) u^{p} .
\end{gathered}
$$

We refer to AE-II, Th. 2 for the proof (in the case where $\gamma=1$ ).

Suppose next that $K=k_{v}$ is a $p$-field, i.e., $v$ is non-archimedean; let $R$ denote the maximal compact subring of $K, P$ its maximal ideal, and $R / P=F_{q}$; let $\pi$ denote an element of $P-P^{2}$ and write an arbitrary element $t$ of $K^{\times}$as $\pi^{e} u$ with $e$ in $Z$ and $u$ in $K_{1}^{\times}=R-P$; then, for every quasicharacter $\omega$ of $K^{\times}$, we have 


$$
\omega(t)=z^{e} \chi(u),
$$

in which $z$ is in $C^{\times}$and $\chi$ is a character of $K_{1}^{\times}$. Conversely, for every $z$ in $C^{\times}$and $\chi$ in the dual of $K_{1}^{\times}$, the above prescription defines a quasicharacter of $K^{\times}$; and the complex power $Z(\omega, \Phi)$ becomes a rational function of $z$ provided that $\operatorname{char}(k)=0$. If we write

$$
Z(\omega, \Phi) \equiv \sum_{\alpha} \sum_{i=1}^{m_{\alpha}} b_{\alpha, i}(\chi)\left(1-\alpha^{-1} z\right)^{-i} \quad \bmod C\left[z, z^{-1}\right],
$$

we have $|\alpha|>1$ and

$$
\begin{aligned}
F_{\Phi}^{*}\left(\pi^{e} u\right)= & {\left[\left(1-q^{-1}\right)^{-1} \sum_{\alpha, x} \sum_{i=1}^{m_{\alpha}}\left(\begin{array}{c}
i-d-e-e_{\chi}-1 \\
i-1
\end{array}\right) b_{\alpha, i}(\chi) g_{x-1} \alpha^{e} \chi \chi(u)\right.} \\
& \left.+\sum_{\alpha} \sum_{i=1}^{m_{\alpha}}\left(\sum_{j=0}^{\infty}\left(\begin{array}{c}
i-d-e+j-1 \\
i-1
\end{array}\right) \alpha^{-j}\right) b_{\alpha, i}(1)\right] \alpha^{d+e}
\end{aligned}
$$

for all small $e$, in which $d$ is the largest integer such that $\psi_{v}=1$ on $P^{-d}$ and $e_{\chi}$ is the smallest positive integer such that $\chi=1$ on $1+P^{e_{\chi}}$; $g_{x}$ for $\chi \neq 1$ is a complex number of absolute value $q^{-\frac{1}{2} e_{\chi}}$ and $g_{1}=-q^{-1}$. Again we refer to AE-II, Th. 2 for the proof (of an equivalent statement).

About the function $F_{\Phi}$, we have only to know the following: if $k_{v}$ is an $\boldsymbol{R}$-field, $\boldsymbol{F}_{\phi}$ is an infinitely differentiable function on $k_{v}^{\times}$such that $F_{\phi}(i)$ tends to 0 as $|i|_{v} \rightarrow \infty$ more rapidly than any negative power of $|i|_{v}$; if $k_{v}$ is a $p$-field, $F_{\Phi}$ is a locally constant function on $k_{v}^{\times}$with bounded support in $k_{v}$. Moreover, the limit $F_{\phi}(0)$ of $F_{\Phi}(i)$ as $|i|_{v} \rightarrow 0$ exists for every $\Phi$ in $\mathscr{S}\left(X_{v}\right)$ if and only if $F_{\phi}^{*}$ is an $L^{1}$-function on $k_{v}$ (for every $\Phi)$; cf. AE-II, Th. 2.

Finally, we recall that the information about $Z(\omega, \Phi)$ comes from the existence of a "Hironaka resolution" of the singularities of $f$. If $X^{\#}$ is the projective space obtained from $X$ by "adding" a hyperplane at infinity, say $E$, and $\mathscr{I}$ the sheaf of ideals associated with the divisor of zeros of the extension $f^{\#}$ of $f$ to a function on $X^{\#}$, the Hironaka resolution of $(E, \mathscr{I})$ is the one defined by his "Main Theorem II (N)" in [3], p. 176. In the case where $f$ is strongly non-degenerate, without any assumption on the characteristic of $k$, the Hironaka resolution exists and is unique; as a morphism, it is simply the monoidal transformation of $X^{\#}$ with the origin 0 of $X$ as its center; and it is "tame" if char $(k)$ does not divide $\operatorname{deg}(f)$. In particular, the above "provision" can be replaced by this condition. In the following sections, we shall tacitly 
assume that $f$ is strongly non-degenerate; we put $\operatorname{dim}(X)=n$ and $\operatorname{deg}(f)=m \geqq 2$.

2. Asymptotic formulas. We shall first consider the case where $k_{v}$ is an $\boldsymbol{R}$-field and (leaving the ambiguity of a numerical constant) determine the "first term" of the asymptotic expansion of $F_{\Phi}^{*}\left(i^{*}\right)$ as $\left|i^{*}\right|_{v} \rightarrow \infty$; we recall that $\Phi$ is an arbitrary Schwartz function on $X_{v}$.

THEOREM 1. We have

$$
F_{\Phi}^{*}\left(i^{*}\right) \approx c_{v} \Phi(0)\left|i^{*}\right|_{v}^{-n / m}+\cdots
$$

as $\left|i^{*}\right|_{v} \rightarrow \infty$, in which $c_{v}=c_{0}+c_{1} \operatorname{sgn}\left(i^{*}\right)$ if $k_{v}=\boldsymbol{R} ; c_{0}, c_{1}$, and $c_{v}$ for $k_{v}=C$ are independent of $\Phi$ and $i^{*}$.

Proof. Consider the complex power $Z(\omega, \Phi)$; then it becomes a finite sum of the following four types of integrals:

$$
\begin{aligned}
& \int_{k_{v}} \omega\left(t^{m}\right)|t|_{v}^{n-1} \phi_{1}(t)|d t|_{v}, \quad \int_{k_{v}^{2}} \omega\left(y_{1}^{m} y_{2}\right)\left|y_{1}\right|_{v}^{n-1} \phi_{2}(y)|d y|_{v}, \\
& \int_{k_{v}} \omega(t) \phi_{3}(t)|d t|_{v}, \quad \quad \int_{X_{v}} \omega\left(f_{v}(x)\right) \phi_{4}(x)|d x|_{v},
\end{aligned}
$$

in which all $\phi$ 's are Schwartz functions; of these $\phi_{1}, \phi_{2}, \phi_{3}$ have compact supports; $\operatorname{Supp}\left(\phi_{4}\right)$ does not contain 0 ; and $\phi_{1}(0)=$ const. $\Phi(0), \phi_{2}(0, t)=$ $\Phi(0) \theta(t)$ with the "const." and the $\theta$ both independent of $\Phi$. Therefore, if $\omega(t)=|t|_{v}^{s}\left(|t|^{-1} t\right)^{p}$ for every $t$ in $k_{v}^{\times}$, then the poles of the meromorphic continuation of $Z(\omega, \Phi)$ are among the following sequences in the $s$-plane:

$$
\begin{aligned}
& -n / m-(1 / 2 d m) \text {-times } 0,1,2, \cdots, \\
& -1-(1 / 2 d) \text {-times } 0,1,2, \cdots,
\end{aligned}
$$

in which $d=\frac{1}{2}$ or 1 according as $k_{v}=\boldsymbol{R}$ or $\boldsymbol{C}$. Moreover, the order of a pole $-\lambda$ is at most 2 . For our purpose, we have only to examine the principal parts at those poles which are not smaller than $-n / m$.

In the case where $k_{v}=\boldsymbol{R}$, the principal parts in question are as follows :

(1) $A_{p} \Phi(0)(s+n / m)^{-1}$;

(2) $A_{\lambda, p}^{\prime}(s+\lambda)^{-1}$, where $\lambda$ is a positive integer at most equal to $n / m$ and $A_{\lambda, p}^{\prime} \neq 0$ only if $\lambda \not \equiv p \bmod 2$;

(3) $A_{p}^{\prime \prime} \Phi(0)(s+n / m)^{-2}$ if $n / m$ is an integer, where $A_{p}^{\prime \prime} \neq 0$ implies $n / m \not \equiv p \bmod 2$. 
Moreover $A_{p}$ in (1) and $A_{p}^{\prime \prime}$ in (3) are independent of $\Phi$. Each one of these principal parts contributes to the asymptotic expansion of $F_{\Phi}^{*}\left(\gamma^{-1} t\right)$ as $|t|_{v} \rightarrow \infty$, in which $\psi_{v}(t)=\boldsymbol{e}(\gamma t)$. The contribution can be determined by the formula in the previous section: from (1) we get

$$
\left(\frac{1}{2}\right)\left(\sum_{p} A_{p} b_{p}(n / m)(\operatorname{sgn} t)^{p}\right) \Phi(0)|t|_{v}^{-n / m} ;
$$

from (2) we get no contribution because $A_{\lambda, p}^{\prime} \neq 0$ implies $\Gamma\left(\frac{1}{2}(1-\lambda+p)\right)$ $=\infty$, hence

$$
b_{p}(\lambda)=i^{p} \pi^{(1 / 2)(1-2 \lambda)} \Gamma\left(\frac{1}{2}(\lambda+p)\right) / \Gamma\left(\frac{1}{2}(1-\lambda+p)\right)=0 ;
$$

and, for a similar reason, from (3) we only get

$$
-\left(\frac{1}{2}\right)\left(\sum_{p} A_{p}^{\prime \prime}\left(d b_{p}(s) / d s\right)_{s=n / m}(\operatorname{sgn} t)^{p}\right) \Phi(0)|t|_{v}^{-n / m}
$$

This implies our theorem for $k_{v}=\boldsymbol{R}$.

In the case where $k_{v}=C$, the principal parts in question are as follows :

(1) $A_{p} \Phi(0)(s+n / m)^{-1}$, where $A_{p} \neq 0$ implies $p=0$;

(2) $A_{\lambda, p}^{\prime}(s+\lambda)^{-1}$, where $\lambda=1+\frac{1}{2}|p|+i$ for some non-negative integer $i$ such that $\lambda$ is at most equal to $n / m$;

(3) $A_{p}^{\prime \prime} \Phi(0)(s+n / m)^{-2}$ if $n / m$ is an integer, and $A_{p}^{\prime \prime} \neq 0$ implies $p=0$.

Moreover $A_{p}$ in (1) and $A_{p}^{\prime \prime}$ in (3) are independent of $\Phi$. Each one of these principal parts contributes to the asymptotic expansion of $F_{\phi}^{*}\left(\gamma^{-1} t\right)$ as $|t|_{v} \rightarrow \infty$, in which $\psi_{v}(t)=e(2 \operatorname{Re}(\gamma t))$ : from (1) we get

$$
(1 / 2 \pi) A_{0} b_{0}(n / m) \Phi(0)|t|_{v}^{-n / m} ;
$$

from (2) we get no contribution (as in the previous case); and from (3) we only get

$$
-(1 / 2 \pi) A_{0}^{\prime \prime}\left(d b_{0}(s) / d s\right)_{s=n / m} \Phi(0)|t|_{v}^{-n / m} .
$$

This implies our theorem for $k_{v}=C$. q.e.d.

Remark. The constants $c_{0}, c_{1}$ for $k_{v}=R$ and $c_{v}$ for $k_{v}=C$ have the following properties: $c_{0}, c_{v}$ are real and $c_{1}$ is pure imaginary; and $c_{1}=0$ if $m$ is odd. These properties can be proved in two ways. One way is to make the above proof more precise: we observe that the "const." 
in $\phi_{1}(0)=$ const. $\Phi(0)$ and $\theta(t)$ in $\phi_{2}(0, t)=\Phi(0) \theta(t)$ are real. This implies that $A_{p}$ and $A_{p}^{\prime \prime}$ are real. Moreover, in the case where $k_{v}=\boldsymbol{R}$, we get $m p \equiv 0 \bmod 2$ from $A_{p} \neq 0$ and also from $A_{p}^{\prime \prime} \neq 0$. The properties of $c_{0}, c_{1}$, and $c_{v}$ follow immediately from these. Another way is simply to manipulate the asymptotic formula in the theorem: we observe that the complex conjugation applied to $F_{\Phi}^{*}\left(i^{*}\right)$ has the effect of replacing $\left(\Phi, i^{*}\right)$ by $\left(\bar{\Phi},-i^{*}\right)$. If $k_{v}=C$, the asymptotic formula as $\left|i^{*}\right|_{v} \rightarrow \infty$ of the complex conjugate of $F_{\phi}^{*}\left(i^{*}\right)$ can be obtained in two different ways; and we get $\bar{c}_{v}=c_{v}$. If $k_{v}=\boldsymbol{R}$, we similarly get $\bar{c}_{0}+\bar{c}_{1}=c_{0}-c_{1}$; this implies that $c_{0}$ is real and $c_{1}$ pure imaginary. In the integral defining $F_{\Phi}^{*}\left(i^{*}\right)$, we replace $x$ by $-x$; then $F_{\Phi}^{*}\left(i^{*}\right)$ and $\Phi(0)$ remain unchanged. If $m$ is odd, however, this has the effect of changing the sign of $i^{*}$. Therefore, by passing to the asymptotic formula, we get $c_{1}=0$.

We shall consider the case where $K=k_{v}$ is a $p$-field and determine $F_{\infty}^{*}\left(i^{*}\right)$ for all large $\left|i^{*}\right|_{v}$; we recall that $\Phi$ is an arbitrary locally constant function with compact support on $X_{v}$.

THEOREM 2. We have

$$
F_{\phi}^{*}\left(\pi^{e} u\right)=\left[\sum_{\alpha^{m}=q^{n}}\left(\sum_{\chi^{m}=1} c_{\alpha, \chi} \chi(u)\right) \alpha^{e}\right] \Phi(0)
$$

for all small $e$ in $Z$ and for every $u$ in $K_{1}^{\times}$, in which $c_{\alpha, x}$ are certain constants independent of $\Phi$ and $i^{*}=\pi^{e} u$.

Proof. We proceed as in the proof of Th. 1: in the non-archimedean case, we only have the first three types of integrals where $\phi$ 's are locally constant functions with compact support; and $\phi_{1}(0)=$ const. $\Phi(0), \phi_{2}(0, t)$ $=\Phi(0) \theta(t)$. Therefore, if $\omega\left(\pi^{e} u\right)=z^{e} \chi(u)$ for every $e$ in $Z$ and $u$ in $K_{1}^{\times}$, we get

$$
\begin{aligned}
Z(\omega, \Phi) \equiv & \sum_{\alpha^{m}=q^{n}} A_{\alpha, \chi} \Phi(0)\left(1-\alpha^{-1} z\right)^{-1}+A^{\prime}\left(1-q^{-1} z\right)^{-1} \\
& +A^{\prime \prime} \delta_{m n} \Phi(0)\left(1-q^{-1} z\right)^{-2} \quad \bmod C\left[z, z^{-1}\right]
\end{aligned}
$$

in which $A_{\alpha, x}, A^{\prime}, A^{\prime \prime}$ are constants and $\delta_{m n}$ is the Kronecker delta; $A_{\alpha, \chi}, A^{\prime \prime}$ are independent of $\Phi ; A_{\alpha, \chi}=0$ unless $\chi^{m}=1$ and $A^{\prime}=A^{\prime \prime}=0$ unless $\chi=1$. By applying the formula we recalled in the previous section, we get 


$$
\begin{aligned}
F_{\phi}^{*}\left(\pi^{e} u\right)= & {\left[\sum _ { \alpha ^ { m } = q ^ { n } } \left(\left(1-q^{-1}\right)^{-1} \sum_{\chi^{m}=1} A_{\alpha, \alpha} g_{\chi^{-1}} \alpha^{e} \chi \chi(u)\right.\right.} \\
& \left.\left.+\left(1-\alpha^{-1}\right)^{-1} A_{\alpha, 1}\right) \alpha^{d+e}+\left(1-q^{-1}\right)^{-2} A^{\prime \prime} \delta_{m n} q^{d+e}\right] \Phi(0)
\end{aligned}
$$

for all small $e$. This can be rewritten as stated in the theorem. q.e.d.

Th. 1 and Th. 2 have the following implication:

COROLlary. We have

$$
\left|F_{\Phi}^{*}\left(i^{*}\right)\right| \leqq \text { const. } \max \left(1,\left|i^{*}\right|_{v}\right)^{-n / m}
$$

for every $i^{*}$ in $k_{v}$, in which the "const." depends on $\Phi$ but not on $i^{*}$.

We might mention that such an estimate was difficult to obtain in the archimedean case. In fact, even for a special $f$, known estimates are considerably less precise than ours.

3. Asymptotic formulas ("good case"). We have introduced coordinates in $X$ with respect to a $k$-base of $X_{k}$. In terms of the coordinates $\left(x_{1}, \cdots, x_{n}\right)$ of $x, f(x)$ becomes a homogeneous polynomial of degree $m$ with coefficients in $k$. Since $f$ is strongly non-degenerate, there exist a positive integer $\rho$ and $n^{2}$ polynomials $A_{i j}(x)$ in $k\left[x_{1}, \cdots, x_{n}\right]$ satisfying

$$
x_{i}^{\rho}=\sum_{j=1}^{n} A_{i j}(x) f_{j}(x)
$$

for $1 \leqq i \leqq n$, in which $f_{j}(x)=\partial f / \partial x_{j}$. For a non-archimedean valuation $v$ on $k$, we have started using the notation $K=k_{v}, R, P$, etc.; we shall use the notation $R^{(n)}$, instead of $R^{n}$, to denote $R \times \cdots \times R$; we similarly define $P^{(n)}$ etc. Also we shall denote by $X_{v}^{\circ}$ the $R^{(n)}$ considered as a (compact open) subset of $X_{v}$. We choose $v$ so that the coefficients of $f(x)$ and $A_{i j}(x)$ for all $i, j$ are in $R$ and $d=0$, i.e., $\psi_{v}=1$ on $R$ but not on $P^{-1}$. We have excluded only a finite number of valuations on $k$ and achieved the following: (1) $f(x)$ is in $R\left[x_{1}, \cdots, x_{n}\right]$; (2) if $\bar{f}(x)$ is the element of $\boldsymbol{F}_{q}\left[x_{1}, \cdots, x_{n}\right]$ obtained from $f(x)$ by reducing its coefficients modulo $P$ and $\bar{a}_{1}, \cdots, \bar{a}_{n}$ elements of any extension field of $\boldsymbol{F}_{q}$ not all 0 , at least one $\bar{f}_{i}(x)=\partial \bar{f}(x) / \partial x_{i}$ does not vanish at $\bar{a}=\left(\bar{a}_{1}, \cdots, \bar{a}_{n}\right)$; (3) $X_{v}^{\circ}$ is of measure 1.

We shall assume that $v$ is such a "good valuation" on $k$; and, for the sake of completeness, we shall prove the following two elementary lemmas : 
LEMMA 1. Let $t$ denote an element of $R$ of order $r$ and $N_{e}(t)$ the number of a $\bmod P^{e}$, where $a$ is in $R^{(n)}$, satisfying $f(a) \equiv t \bmod P^{e}$; then, if $e>r$, we have

$$
\begin{aligned}
q^{-(n-1) e} N_{e}(t)= & \left(\sum_{0 \leqq m i<r} q^{-(n-m) i}\right) q^{-(n-1)}\left(N_{1}(0)-1\right) \\
& +\delta_{r 0}^{\prime} q^{-(n / m-1) r-(n-1)} N_{1}\left(\pi^{-r} t\right)
\end{aligned}
$$

in which $\delta_{r 0}^{\prime}=1$ or 0 according as $r \equiv 0$ or $r \not \equiv 0 \bmod m$; if $r=\infty$, i.e., if $t=0$, we have

$$
q^{-(n-1) e} N_{e}(0)=q^{e+n[-e / m]}+\left(\sum_{0 \leqq m i<e} q^{-(n-m) i}\right) q^{-(n-1)}\left(N_{1}(0)-1\right),
$$

in which [ ] is the Gauss symbol.

Proof. Suppose first that $t \neq 0$. If $f(a) \equiv t \bmod P^{e}, a \equiv 0 \bmod P^{i}$, and $e>r$, then we get $m i \leqq r$; hence

$$
N_{e}(t)=\sum_{0 \leqq m i \leqq r} q^{(m-1) n i} \cdot \text { card },
$$

where the "card" is the number of $a \bmod P^{e-m i}$ satisfying

$$
f(a) \equiv \pi^{-m i} t \bmod P^{e-m i}, \quad a \neq \equiv \bmod P .
$$

By the usual lifting process of a solution $\bmod P$ to solutions modulo higher powers of $P$, we get

$$
" \text { card } "=\left\{\begin{array}{l}
\left(N_{1}(0)-1\right) q^{(n-1)(e-m i-1)} \\
N_{1}\left(\pi^{-r} t\right) q^{(n-1)(e-m i-1)}
\end{array}\right.
$$

according as $m i<r$ or $m i=r$; the rest is clear.

Suppose next that $t=0$. If $a \equiv 0 \bmod P^{i}$ and $m i \geqq e$, we obviously have $f(a) \equiv 0 \bmod P^{e}$; hence

$$
N_{e}(0)=1+\sum_{e \leqq m i<m e}\left(1-q^{-n}\right) q^{n(e-i)}+\sum_{0 \leqq m i<e} q^{(m-1) n i} \cdot \text { card },
$$

where the "card" is the number of $a \bmod P^{e-m i}$ satisfying $f(a) \equiv 0$ $\bmod P^{e-m i}, a \neq 0 \bmod P$. Hence, by the usual lifting process, we get

$$
\text { "card" }=\left(N_{1}(0)-1\right) q^{(n-1)(e-m i-1)} ;
$$

the rest is straightforward. q.e.d.

LEMMA 2. Let $\Phi$ denote the characteristic function of $X_{v}^{\circ}$; then for every $i^{*}$ in $K^{\times}$of order $-e \leqq 0$, we have 


$$
F_{\Phi}^{*}\left(i^{*}\right)=\left\{\begin{array}{l}
q^{n[-e / m]} \\
q^{-n((e-1) / m+1)} \sum_{t \bmod P} \psi_{v}\left(\pi^{e-1} i^{*} t\right) N_{1}(t)
\end{array}\right.
$$

according as $e \neq \equiv 1$ or $e \equiv 1 \bmod m$.

Proof. By Lemma 1 and by the orthogonality of characters (of a finite commutative group), we get

$$
\begin{aligned}
F_{\Phi}^{*}\left(i^{*}\right)= & \int_{X_{v}^{o}} \psi_{v}\left(f(x) i^{*}\right)|d x|_{v} \\
= & \sum_{t \bmod P e} \psi_{v}\left(t i^{*}\right) q^{-n e} N_{e}(t) \\
= & q^{-e}\left(q^{e+n[-e / m]}\right. \\
& +\left(\sum_{e-1 \leqq m i<e} q^{-(n-m) i}\right) q^{-(n-1)}\left(N_{1}(0)-1\right) \\
& \left.+\delta_{e-1,0}^{\prime} q^{-(n / m-1)(e-1)-n+1} \sum^{\prime} \psi_{v}\left(t i^{*}\right) N_{1}\left(\pi^{-(e-1)} t\right)\right),
\end{aligned}
$$

where the summation $\Sigma^{\prime}$ is relative to $t \bmod P^{e}$ satisfying ord $(t)=e-1$. The rest is straightforward. q.e.d.

In the notation of Lemma 2 , we have

$$
\left|F_{\phi}^{*}\left(i^{*}\right)\right| \leqq\left|i^{*}\right|_{v}^{-n / m}
$$

for every $i^{*}$ in $K-P$ provided that $e=-$ ord $\left(i^{*}\right) \not \equiv 1 \bmod m$; we shall examine the case where $e \equiv 1 \bmod m$ : we change our notation slightly and denote by $t$ an element of $\boldsymbol{F}_{q}$, by $\psi$ any non-trivial character of $\boldsymbol{F}_{q}$, and by $N(t)$ the number of solutions of $\bar{f}(x)-t=0$ in $\boldsymbol{F}_{q}^{n}$. Then by Lemma 2 we can rewrite $\left(^{*}\right)$ as

$$
\left|q^{-n} \sum_{t} \psi(t) N(t)\right| \leqq q^{-n / m},
$$

in which the summation is taken over $F_{q}$; it is equal to the sum of $\psi(\bar{f}(a))$ for $a$ running over $\boldsymbol{F}_{q}^{n}$. In the special case where $m=2$, it is well known (and easy to show) that $(* *)$ holds with the equality sign. On the other hand, if $n \geqq 3$, then $\bar{f}(x)-t x_{0}^{m}$ is absolutely irreducible for every $t$ in $\boldsymbol{F}_{q}$ and $\bar{f}(x)-t x_{0}^{m}=0$ defines a non-singular projective hypersurface over $\boldsymbol{F}_{q}$. Therefore we can apply the "Riemann-Weil hypothesis" proved recently (after the works of Grothendieck and others) by Deligne [1]; see also Dwork [2]:

In the above notation, "there exist complex numbers $\alpha_{1}, \alpha_{2}, \cdots$ of absolute value $q^{(1 / 2) n-1}$ such that 


$$
N(0)=q^{n-1}+(-1)^{n}(q-1) \sum_{i} \alpha_{i} ;
$$

also there exist complex numbers $\alpha_{1}(t), \alpha_{2}(t), \cdots$ of absolute value $q^{(1 / 2)(n-1)}$ depending on $t \neq 0$ such that

$$
N(t)=q^{n-1}-(-1)^{n}\left(\sum_{i} \alpha_{i}+\sum_{j} \alpha_{j}(t)\right)
$$

the number of $\alpha_{i}$ 's and the number of $\alpha_{j}(t)$ 's depend only on $m$ and $n$." If we use this result, we get

$$
q^{-n} \sum_{t} \psi(t) N(t)=(-1)^{n} q^{-n}\left(q \sum_{i} \alpha_{i}-\sum_{t \neq 0} \psi(t)\left(\sum_{\jmath} \alpha_{j}(t)\right)\right),
$$

hence

$$
\left|q^{-n} \sum_{t} \psi(t) N(t)\right| \leqq c \cdot q^{-(1 / 2)(n-1)}
$$

for some constant $c$ depending only on $m$ and $n$. Since we have $\frac{1}{2}(n-1)$ $-n / m \geqq 1 / 6$ for $n>m \geqq 3$, the inequality:

$$
c \cdot q^{-(1 / 2)(n-1)}>q^{-n / m}
$$

implies $c^{6}>q$; the number of such $v^{\prime}$ 's is finite. We have thus obtained the following theorem:

THEOREM 3. Suppose that $n>m$, i.e., $\operatorname{dim}(X)>\operatorname{deg}(f)$; then there exists a finite set $S$ of valuations on $k$ containing the set $S_{\infty}$ of archimedean valuations such that if $v$ is not in $S$ and $\Phi$ is the characteristic function of $X_{v}^{\circ}$, we have

$$
\left|F_{\Phi}^{*}\left(i^{*}\right)\right| \leqq \max \left(1,\left|i^{*}\right|_{v}\right)^{-n / m}
$$

for every $i^{*}$ in $k_{v}$.

We recall that we have the standing hypothesis that $f$ is strongly non-degenerate and $m \geqq 2$.

4. A tempered measure on $X_{A}$. We shall start from a local consideration; the following lemma follows from what we have reviewed in $\S 1$, from the corollary in $\S 2$ (of Th. 1 and Th. 2), and from AE-II, Th. 3:

LEMma 3. Suppose that $n>m$; then the image measure of $\left|\theta_{0}\right|_{v}$ under the inclusion $U(0)_{v} \rightarrow X_{v}$ is tempered and 


$$
F_{\Phi_{v}}(i)=\int_{X_{v}} \Phi_{v}(x)\left|\theta_{i}(x)\right|_{v}
$$

defines a continuous $L^{1}$-function $F_{\Phi_{v}}$ on $k_{v}$ for every $\Phi_{v}$ in $\mathscr{S}\left(X_{v}\right)$. Moreover its Fourier transform $F_{\Phi_{v}}^{*}$ is also a continuous $L^{1}$-function on $k_{v}$; and we have

$$
F_{\Phi_{v}}(i)=\int_{k_{v}} F_{\Phi_{v}}^{*}\left(i^{*}\right) \psi_{v}\left(-i i^{*}\right)\left|d i^{*}\right|_{v}
$$

for every $i$ in $k_{v}$.

For every $i$ in $k_{v}$, we have recalled the definition of $U(i)_{v}$ in $\S 1$. Suppose that $v$ is a good valuation. If $i$ is $v$-integral and different from 0 , we define $U(i)_{v}^{\circ}$ as the (compact open) subset of $U(i)_{v}$ consisting of $v$-integral points; if $i=0$, we define $U(0)_{v}^{\circ}$ as the subset of $U(0)_{v}$ consisting of $v$-primitive points, i.e., $v$-integral points with $v$-units among their coordinates. Then, in the notation of the previous section, we have

$$
\left\{\begin{array}{l}
\int_{U(i)_{v}^{\circ}}\left|\theta_{i}\right|_{v}=q^{-(n-1)} N(\bar{i}) \\
\int_{U(0){ }_{v}}\left|\theta_{0}\right|_{v}=q^{-(n-1)}(N(0)-1)
\end{array}\right.
$$

provided that $\bar{i}$, the residue class of $i \bmod P$, is different from 0 . We can apply Deligne's result to the right hand sides; and we get

$$
\left.\left|\int_{U(i) \stackrel{v}{v}}\right| \theta_{i}\right|_{v}-1 \mid \leqq c \cdot q^{-(1 / 2)(n-1)}, c \cdot q^{-(1 / 2) n+1}
$$

according to the cases, in which $c$ is a constant independent of $v$; the upper bound $c \cdot q^{-(1 / 2) n+1}$ can obviously be used in both cases.

We shall change our notation slightly and denote by $i$ an element of $k$; then the above estimates hold for almost all $v$. Consider the following infinite product:

$$
\prod_{v}^{\prime} \int_{U(i))_{v}^{v}}\left|\theta_{i}\right|_{v}
$$

extended over the set of good valuations or over its subset defined by $|i|_{v}=1$ according as $i=0$ or $i \neq 0$. Then, if $\frac{1}{2} n-1>1$, i.e., if $n>4$, it is convergent (in the usual sense if we exclude a finite number of factors which may be 0 ). Therefore the restricted product measure 
$\left|\theta_{i}\right|_{A}$ of all $\left|\theta_{i}\right|_{v}$ is defined on the adelization $U(i)_{A}$ of $U(i)$ provided that $n>4$; this provision can be replaced by $n>3$ for $i \neq 0$. Moreover the image measure of $\left|\theta_{i}\right|_{A}$ under $U(i)_{A} \rightarrow X_{A}$ is tempered for every $i \neq 0$; by Lemma 3 it is also tempered for $i=0$ if $n>m$. Therefore, if $n>$ $\max (m, 4)$, then

$$
F_{\Phi}(i)=\int_{U(i)_{A}} \Phi(x)\left|\theta_{i}(x)\right|_{A}
$$

is defined for every $\Phi$ in $\mathscr{S}\left(X_{A}\right)$ and $i$ in $k$.

THEOREM 4. Suppose that $n>\max (m+1,4)$ and let $C$ denote $a$ compact subset of $\mathscr{S}\left(X_{A}\right)$; then the series

$$
\sum_{i \in k} F_{\emptyset}(i)
$$

for $\Phi$ varying in $C$ has a "dominant series."

Proof. There exists a finite set $S$ of valuations on $k$ containing $S_{\infty}$ and an element $\phi_{v} \geqq 0$ of $\mathscr{S}\left(X_{v}\right)$ for each $v$, which is equal to the characteristic function of $X_{v}^{\circ}$ for every $v$ not in $S$, such that

$$
|\Phi(x)| \leqq \phi(x)=\left(\prod_{v} \phi_{v}\right)(x)
$$

for every $\Phi$ in $C$ and $x$ in $X_{A}$. For the proof, we refer to [8], Lemma 5 ; see also [5], Lemma 7. We may assume that every $v$ not in $S$ is a good valuation. We have only to show that the series of $F_{\phi}(i)$ for $i$ running over $k^{\times}$is convergent.

First of all, we have

$$
F_{\phi}(i)=\prod_{v} F_{\phi_{v}}(i)
$$

for every $i$. We observe that if $v$ is not in $S_{\infty}$, the image of $\operatorname{Supp}\left(\phi_{v}\right)$ under $f_{v}$ is compact. Therefore the set of all $i$ for which $F_{\phi_{v}}(i) \neq 0$ is bounded in $k_{v}$. In particular, if $v$ is not in $S$, then $F_{\phi_{v}}(i) \neq 0$ implies $|i|_{v} \leqq 1$. Since $F_{\phi_{v}}(i)=0$ implies $F_{\phi}(i)=0$, we may restrict $i$ in $k^{\times}$by the condition that $|i|_{v} \leqq 1$ for every $v$ not in $S$ and $|i|_{v} \leqq$ const. for every $v$ in $S-S_{\infty}$. Then, in the case where $k$ is a function field, we just get a finite set; hence there is no problem. Therefore we may assume that $k$ is a number field. In that case, $i$ is contained in a fractional ideal of $k$; and its image in 


$$
k_{\infty}=k \underset{Q}{\otimes} \boldsymbol{R}=\prod_{v \in S_{\infty}} k_{v}
$$

is a lattice in this vector space.

If $v$ is not in $S$ and $0<|i|_{v} \leqq 1$, by Lemma 1 we have

$$
F_{\phi_{v}}(i)=q^{-(n-1) e} N_{e}(i)
$$

for any $e>\operatorname{ord}_{v}(i)$. Therefore $F_{\phi_{v}}(i)$ is equal to $q^{-(n-1)} N_{1}(i)$ if $\operatorname{ord}_{v}(i)$ $=0$; it has

$$
\left(1-q^{-(n-m)}\right)^{-1} q^{-(n-1)} N_{1}(0)+q^{-(n-m)-(n-1)} \max _{\mid t_{b}=1} N_{1}(t)
$$

as an upper bound if $\operatorname{ord}_{v}(i)>0$ provided that $n>m$. We evaluate these further by Deligne's result and we get

$$
F_{\phi_{v}}(i) \leqq\left(1-2 q^{-2}\right)^{-1}\left(1+c q^{-(1 / 2) n+1}\right)
$$

for some constant $c$ independent of $v$ and $i$ provided that $n>m+1$. Therefore, if $n>\max (m+1,4)$, we get

$$
\prod_{v \notin S} F_{\phi_{v}}(i) \leqq c_{1}
$$

for some constant $c_{1}$ independent of $i$.

On the other hand, if $n>m$, then $F_{\phi_{v}}$ is a continuous function on $k_{v}$ for every $v$; it has a compact support for $v$ not in $S_{\infty}$; and $F_{\phi_{v}}\left(i_{v}\right)$ tends to 0 as $\left|i_{v}\right|_{v} \rightarrow \infty$ more rapidly than any negative power of $\left|i_{v}\right|_{v}$ for $v$ in $S_{\infty}$. This follows from Lemma 3 and from what we have reviewed in $\S 1$. Therefore we get

$$
\prod_{v \in S} F_{\phi_{v}}(i) \leqq c_{2} \prod_{v \in S_{\infty}} \max \left(1,|i|_{v}\right)^{-2}
$$

for some constant $c_{2}$ independent of $i$. If we denote the summation over the above-mentioned lattice in $k_{\infty}$ by $\Sigma^{\prime}$, by putting these together, we get

$$
\sum_{i \in k \times} F_{\phi}(i) \leqq c_{1} c_{2} \sum_{i}^{\prime} \prod_{v \in S_{\infty}} \max \left(1,|i|_{v}\right)^{-2} ;
$$

and the right hand side is convergent, say, by [5], Lemma 12. q.e.d.

We recall that each $F_{\Phi}(i)$ is a tempered measure on $X_{A}$. Therefore Th. 4 shows that the series of $F_{\emptyset}(i)$ for $i$ running over $k$ also defines a tempered measure on $X_{A}$. We shall add the following remark: 
Remark. Suppose that $k$ is a number field and denote by $k^{\circ}$ its principal order, by $Q$ an integral ideal of $k$, and by $|Q|$ its absolute norm; assume that $f(x)$ is in $k^{0}\left[x_{1}, \cdots, x_{n}\right]$ and, for every $i \neq 0$ in $k^{o}$, let $N_{Q}(i)$ denote the number of $a \bmod Q$, where $a$ is in $\left(k^{o}\right)^{(n)}$, satisfying $f(a) \equiv i \bmod Q$. Then the limit (if it exists) of $|Q|^{-(n-1)} N_{Q}(i)$ as $Q$ becomes divisible by any given integral ideal of $k$ is called the "singular series" associated with $f(x)$ and $i$; cf. [7]. This is related to $F_{\phi}(i)$ as follows :

We decompose $X_{A}$ into $X_{0} \times X_{\infty}$, where $X_{0}$ is the restricted product of $X_{v}$ for all $v$ not in $S_{\infty}$ and $X_{\infty}$ the product of $X_{v}$ for $v$ in $S_{\infty}$; similarly, we decompose $U(i)_{A}$ into $U(i)_{0} \times U(i)_{\infty}$ and $\left|\theta_{i}\right|_{A}$ into $\left|\theta_{i}\right|_{0} \otimes\left|\theta_{i}\right|_{\infty}$. Let $\Phi_{0}$ denote the characteristic function of the product of $X_{v}^{\circ}$ for all $v$ not in $S_{\infty}, \Phi_{\infty}$ an arbitrary Schwartz function on $X_{\infty}$, and $\Phi=\Phi_{0} \otimes \Phi_{\infty}$. Then we have

$$
F_{\Phi}(i)=\left(\lim _{Q}|Q|^{-(n-1)} N_{Q}(i)\right) \cdot \int_{X_{\infty}} \Phi_{\infty}(x)\left|\theta_{i}(x)\right|_{\infty}
$$

provided that $n \geqq 4$.

5. Poisson formula. We shall turn our attention to another type of functions; we shall first prove the following general lemma:

LEMMA 4. Let $r$ denote a non-negative integer, $\varepsilon$ a positive real number, and $\sigma_{v}$ for each valuation $v$ on $k$ a real number; suppose that $\sigma_{v}>r$ for all $v$ and $\sigma_{v} \geqq r+1+\varepsilon$ for almost all $v$. Then the series

$$
\sum_{i} \prod_{v} \max \left(1,\left|i_{1}\right|_{v}, \cdots,\left|i_{r}\right|_{v}\right)^{-\sigma_{v}}
$$

in which $i=\left(i_{1}, \cdots, i_{r}\right)$ runs over $k^{r}$, is convergent.

Proof. In the case where $k$ is a number field, this lemma was proved in [5] as Prop. 1. Suppose, therefore, that $k$ is a function field of genus $g$; let $\boldsymbol{F}_{q_{0}}$ denote the algebraic closure in $k$ of the prime field. Since $k$ contains a prime divisor of arbitrarily large degree, we can choose one, say $p_{\infty}$, satisfying $\operatorname{deg}\left(p_{\infty}\right) \geqq 2 g$; let $\mid l_{\infty}$ denote the usual absolute value at $p_{\infty}$. For any non-negative integer $e$, let $L\left(e p_{\infty}\right)$ denote the vector space over $\boldsymbol{F}_{q_{0}}$ of elements of $k$ with poles only at $p_{\infty}$ and with orders at most $e$; then $L\left(p_{\infty}\right)$ contains an element $t$ not in $\boldsymbol{F}_{q_{0}}$. 
Let $k^{\circ}$ denote the integral closure of $F_{q_{0}}[t]$ in $k$ and $\lambda, \alpha$ real numbers satisfying $\lambda \geqq 1, \alpha>1$; then we have

$$
\sum_{i \in \kappa^{\circ}} \max \left(\lambda,|i|_{\infty}\right)^{-\alpha} \leqq c \cdot \lambda^{1-\alpha}
$$

for some constant $c$ independent of $\lambda$. This is a counterpart of [5], Lemma 12 ; and it can be proved as follows:

We observe that $k^{\circ}$ is the union of $L\left(e p_{\infty}\right)$ for $e=0,1,2, \cdots$. Moreover, if $q$ denotes the power of $q_{0}$ with $\operatorname{deg}\left(p_{\infty}\right)$ as its exponent, we have $|t|_{\infty}=q$. Therefore the left hand side is equal to

$$
q_{0} \lambda^{-\alpha}+\sum_{e=1}^{\infty} \operatorname{card}\left(L\left(e p_{\infty}\right)-L\left((e-1) p_{\infty}\right)\right) \max \left(\lambda, q^{e}\right)^{-\alpha},
$$

in which $L(0)=F_{q_{0}}$. And by the Riemann-Roch theorem we get

$$
\operatorname{card}\left(L\left(e p_{\infty}\right)\right)=\left(q_{0}\right)^{1-g} q^{e}
$$

for $e=1,2, \ldots$. If we split the above summation into two parts by $q^{e} \leqq \lambda$ and $q^{e}>\lambda$, we can convince ourselves that both parts are of order $\lambda^{1-\alpha}$.

Once we have that, the rest can be proved in the same way as "Prop. 1." In fact, since the group of units of $k^{\circ}$ is $\left(\boldsymbol{F}_{q_{0}}\right)^{\times}$, the proof is simpler. q.e.d.

Let $\Phi$ denote an arbitrary element of $\mathscr{S}\left(X_{A}\right)$ and $C$ a compact subset of $k_{A}$; then, if $n>m$, there exists a finite set $S$ of valuations on $k$ with the following properties: Th. 3 holds for $S$ and every $v$ not in $S$ is a good valuation; $\Phi$ decomposes into the product of the characteristic function of $X_{v}^{\circ}$ for all $v$ not in $S$ and a Schwartz-Bruhat function $\Phi_{S}$ of the product $X_{S}$ of $X_{v}$ for $v$ in $S$; for every $v$ not in $S$, the image of $C$ under the canonical projection $k_{A} \rightarrow k_{v}$ is contained in the unit $\operatorname{disc} R$.

Let $|d x|_{S}$ denote the product measure of $|d x|_{v}$ for all $v$ in $S$; then, by using the corollary in $\S 1$ (of Th. 1 and Th. 2) and Th. 3, we get

$$
\begin{aligned}
\left|F_{\Phi}^{*}\left(i^{*}+i\right)\right| \leqq & \prod_{v \notin S} \max \left(1,|i|_{v}\right)^{-n / m} \\
& \left.\cdot\left|\int_{X_{S}} \Phi_{S}(x) \prod_{v \in S} \psi_{v}\left(f(x)\left(i_{v}^{*}+i\right)\right)\right| d x\right|_{S} \mid \\
\leqq & c \prod_{v} \max \left(1,|i|_{v}\right)^{-n / m}
\end{aligned}
$$


for every $i^{*}=\left(i_{v}^{*}\right)_{v}$ in $C$ and $i$ in $k$, in which the constant $c$ is independent of $i^{*}$ and $i$. Actually we can show that such a constant exists even if we let $\Phi$ vary in a compact subset of $\mathscr{S}\left(X_{A}\right)$. At any rate, by taking $r=1$ and $\sigma_{v}=n / m$ in Lemma 4 , we see that the following series:

$$
\Theta\left(i^{*}\right)=\sum_{i \in k} F_{\Phi}^{*}\left(i^{*}+i\right)
$$

for $i^{*}$ varying in $C$ has a dominant series if $n>2 m$. Since $k_{A} / k$ is compact, we may assume that $k_{A}=k+C$. In this way, we see that the above series defines a continuous function on $k_{A} / k$ and that $F_{D}^{*}$ is a continuous $L^{1}$-function on $k_{A}$. Let

$$
\Theta\left(i^{*}\right)=\sum_{i \in k} a(i) \psi\left(i i^{*}\right)
$$

denote the Fourier expansion of $\Theta\left(i^{*}\right)$; then we get

$$
a(i)=\int_{k_{A}} F_{\Phi}^{*}\left(i^{*}\right) \psi\left(-i i^{*}\right)\left|d i^{*}\right|_{A} .
$$

By applying Lemma 3, we see that the right hand side coincides with $F_{\phi}(i)$ for every $i$; hence

$$
\sum_{i \in k} F_{\phi}(i) \psi\left(i i^{*}\right)=\sum_{i \in k} F_{\phi}^{*}\left(i^{*}+i\right)
$$

for every $i^{*}$ in $k_{A}$ provided that the left hand side is absolutely convergent. Since $n>2 m$ implies $n>\max (m+1,4)$, the absolute convergence is guaranteed by Th. 4. And, by putting $i^{*}=0$, we get the following theorem:

THEOREM 5. Suppose that $n>2 m$, i.e., $\operatorname{dim}(X)>2 \operatorname{deg}(f)$; then for every $\Phi$ in $\mathscr{S}\left(X_{A}\right)$ we have

$$
\sum_{i \in k} \int_{U(i) A} \Phi(x)\left|\theta_{i}(x)\right|_{A}=\sum_{i^{*} \in k} \int_{X_{A}} \Phi(x) \psi\left(f(x) i^{*}\right)|d x|_{A},
$$

in which both sides are absolutely convergent.

We observe that the integrand $\Phi_{i^{*}}(x)=\Phi(x) \psi\left(f(x) i^{*}\right)$ on the right hand side is in $\mathscr{S}\left(X_{A}\right)$ and that the above tempered measure on $X_{A}$ takes the same value at $\Phi(x)$ and $\Phi_{i *}(x)$. The correspondence $\Phi \rightarrow \Phi_{i^{*}}$ uniquely extends to a unitary operator on $L^{2}\left(X_{A}\right)$. This invariance property is preserved even if we introduce a tempered measure $E(\Phi)$ as 


$$
E(\Phi)=\sum_{i^{*} \in k} \int_{X_{A}} \Phi(x) \psi\left(f(x) i^{*}\right)|d x|_{A}+\Phi(0) .
$$

In the special case where $m=2$, if we put $f(x, y)=f(x+y)-f(x)-$ $f(y)$, the new measure $E(\Phi)$ is also invariant under the following twisted Fourier transformation:

$$
\hat{\Phi}(x)=\int_{X_{A}} \Phi(y) \psi(f(x, y))|d y|_{A}
$$

cf. [9], p. 64. In this way, we see that $E(\Phi)$ is invariant under a group of unitary operators which is isomorphic to $S L_{2}(k)$. It is an interesting problem to examine whether this classical result has a generalization of some kind to the case where $m \geqq 3$.

6. The constants $i_{v}\left(\psi_{v} \circ f\right)$. We shall add some remarks on the numerical constants which appeared in the asymptotic formulas for $F_{\phi}^{*}\left(i^{*}\right)$ as $\left|i^{*}\right|_{v} \rightarrow \infty$ : consider $\psi_{v}(f(x))$ as a tempered distribution $T(x)=$ $T_{v}(x)$ on $X_{v}$ as

$$
T[\Phi]=\int_{X_{v}} \Phi(x) \psi_{v}(f(x))|d x|_{v}
$$

for every $\Phi$ in $\mathscr{S}\left(X_{v}\right)$; let $T^{*}$ denote its Fourier transform defined by $T^{*}[\Phi]=T\left[\Phi^{*}\right]$, where

$$
\Phi^{*}(x)=\int_{X_{v}} \Phi(y) \psi_{v}([x, y])|d y|_{v} .
$$

Then $T^{*}$ is an analytic function on $X_{v}$ for $k_{v}=R, C$. We have learned this fact from L. Ehrenpreis; it can be proved as follows:

Suppose first that $k_{v}=R$; for our purpose, we may assume that $\psi_{v}(t)=\boldsymbol{e}(t)$ for every $t$ in $\boldsymbol{R}$. If $P(\xi)$ is a polynomial in $n$ letters $\xi_{1}, \cdots, \xi_{n}$ with complex coefficients, we shall denote by $P(\partial / \partial x)$ the differential operator obtained from $P(\xi)$ by replacing each $\xi_{i}$ by $\partial / \partial x_{i}$; as before, we put $f_{i}(x)=\partial f / \partial x_{i}$ for $1 \leqq i \leqq n$. We observe that the distribution $T^{*}$ on $X_{v}=\boldsymbol{R}^{n}$ satisfies

$$
f_{i}(\partial / \partial x) T^{*}+\left(2 \pi(-1)^{1 / 2}\right)^{m-1} x_{i} T^{*}=0
$$

for $1 \leqq i \leqq n$, in which $m=\operatorname{deg}(f) \geqq 2$. Therefore the analyticity of $T^{*}$ follows from the following theorem:

"Let $P_{1}(\xi), P_{2}(\xi), \cdots$ denote a finite number of homogeneous polynomials of the same positive degree in $\xi_{1}, \cdots, \xi_{n}$ with real coefficients 
such that they do not vanish simultaneously at any point of $\boldsymbol{R}^{n}-\{0\}$; let $D_{1}, D_{2}, \cdots$ denote linear partial differential operators of the form

$$
D_{i}=P_{i}(\partial / \partial x)+\text { lower terms, }
$$

in which the coefficients of the lower terms are analytic on some open subset $U$ of $\boldsymbol{R}^{n}$. Then any distribution $S$ on $U$ satisfying $D_{i} S=0$ for every $i$ is analytic on $U . "$ (This theorem itself follows from the standard theorem in, e.g., [4], p. 178 by observing that

$$
D=\sum_{i} P_{i}(\partial / \partial x) D_{i}
$$

is "elliptic" and $D S=0$.)

Suppose next that $k_{v}=C$; let $\left(z_{1}, \cdots, z_{n}\right)$, instead of $\left(x_{1}, \cdots, x_{n}\right)$, denote coordinates on $X_{v}$; put $x_{i}=\operatorname{Re}\left(z_{i}\right), y_{i}=\operatorname{Im}\left(z_{i}\right)$, and $F(x, y)=$ $2 \operatorname{Re}\left(f_{v}(z)\right)$; then $F(x, y)$ has the same property as the $f_{v}(x)$ for $k_{v}=\boldsymbol{R}$ : it is a homogeneous polynomial of degree $m$ in $x_{1}, \cdots, x_{n}, y_{1}, \cdots, y_{n}$ with real coefficients such that its $2 n$ partial derivatives do not vanish simultaneously at any point of $R^{2 n}-\{0\}$. Therefore $T^{*}$ is analytic on (the underlying real vector space of) $X_{v}=C^{n}$.

THEOREM 6. In the notation of Th. 1, we have $T_{v}^{*}(0)=c_{0}+c_{1}$ or $c_{v}$ according as $k_{v}=\boldsymbol{R}$ or $\boldsymbol{C}$.

Proof. We have

$$
c_{v} \Phi(0)=\lim _{t \rightarrow \infty}|t|_{v}^{n} F_{\Phi}^{*}\left(t^{m}\right)
$$

for every $\Phi$ in $\mathscr{S}\left(X_{v}\right)$. We choose an element of $\mathscr{S}\left(X_{v}\right)$ with compact support; call it $\phi$ and take its Fourier transform $\phi^{*}$ as $\Phi$; then we get

$$
\begin{aligned}
c_{v} \phi^{*}(0) & =\lim _{t \rightarrow \infty} \int_{X_{v}} \phi(x) T^{*}\left(t^{-1} x\right)|d x|_{v} \\
& =T^{*}(0) \int_{X_{v}} \phi(x)|d x|_{v} .
\end{aligned}
$$

We have used the fact that $T^{*}\left(t^{-1} x\right)$ tends uniformly to $T^{*}(0)$ on the compact subset $\operatorname{Supp}(\phi)$ of $X_{v}$. Since $\phi$ is an arbitrary element of $\mathscr{S}\left(X_{v}\right)$ with compact support, we get $c_{v}=T^{*}(0)$. q.e.d.

We shall consider the case where $K=k_{v}$ is a $p$-field and prove the following counterpart of Th. 6: 
THEOREM 7. The constant $T_{v}^{*}(0)$ is meaningful; and, in the notation of Th. 2, we have

$$
T_{v}^{*}(0)=\sum_{\alpha^{m}=q^{n}}\left(\sum_{\chi^{m}=1} c_{\alpha, \chi} \chi(1)\right)
$$

Proof. As a tempered distribution on $X_{v}, T^{*}$ is a finitely additive function on the family of all compact open subsets of $X_{v}$. Let $e$ denote a non-negative integer and $\phi_{e}$ the characteristic function of $\left(P^{e}\right)^{(n)}$; then the integral of $\phi_{e}$ over $X_{v}$ is $q^{-((1 / 2) d+e) n}$ and, if $\Phi=\phi_{0}$, we have

$$
\lim _{e \rightarrow \infty} q^{((1 / 2) d+e) n} T^{*}\left[\phi_{e}\right]=\lim _{e \rightarrow \infty} q^{(d+e) n} F_{\Phi}^{*}\left(\pi^{-(d+e) m}\right) .
$$

According to Th. 2, the expression on the right hand side (under the limit sign) is equal to

$$
\sum_{\alpha^{m}=q^{n}}\left(\sum_{\chi^{m}=1} c_{\alpha, \chi} \chi(1)\right)
$$

for all large $e$. We observe that this finite sum does not depend on the choice of the coordinates by which the sequence $\phi_{0}, \phi_{1}, \ldots$ is defined. Therefore we may call the above limit the "derivative" of $T^{*}$ at 0 and denote it by $T^{*}(0)$. q.e.d.

We shall change our notation and denote $T_{v}^{*}(0)$ by $i_{v}\left(\psi_{v} \circ f\right)$ for every $v$. Suppose that $v$ is a good valuation and $a_{v} \neq 0$ an element of $K=k_{v}$ of order $e_{0}$; then we get

$$
i_{v}\left(\psi_{v} \circ a_{v} f\right)=\left\{\begin{array}{l}
q^{n\left[e_{0} / m\right]} \\
q^{n\left(\left(e_{0}+1\right) / m-1\right)} \sum_{t \bmod P} \psi_{v}\left(\pi^{-e_{0}-1} a t\right) N_{1}(t)
\end{array}\right.
$$

according as $e_{0} \not \equiv-1$ or $e_{0} \equiv-1 \bmod m$. This can be derived from Lemma 2. In particular, by taking $a_{v}=1$, we get $i_{v}\left(\psi_{v} \circ f\right)=1$. Therefore we can define $i(\psi \circ f)$ as

$$
i(\psi \circ f)=\prod_{v} i_{v}\left(\psi_{v} \circ f\right)
$$

the product on the right hand side is really a finite product. In the case where $m=2, i_{v}\left(\psi_{v} \circ f\right)$ is equal to Weil's $\gamma_{v}\left(\psi_{v} \circ f\right)$ except for the square root of the absolute value in $k_{v}$ of the discriminant of $f$; cf. [8], p. 161. We recall that the product of $\gamma_{v}\left(\psi_{v} \circ f\right)$ is 1 ; cf. op. cit., p. 179. By an elementary product formula, the product of the absolute 
value in $k_{v}$ of the discriminant of $f$ is 1 ; hence we get $i(\psi \circ f)=1$. We shall give an example indicating that a theorem concerning $i(\psi \circ f)$ (even if it exists) can not have such a simple form for $m \geqq 3$.

EXAMPLE. We take $k=\boldsymbol{Q}, \psi_{\infty}(t)=\boldsymbol{e}(-t)$ for every $t$ in $\boldsymbol{Q}_{\infty}=\boldsymbol{R}$, $\psi_{p}(t)=e(\langle t\rangle)$ for every $t$ in $Q_{p}$, where $\langle t\rangle$ denotes the "fractional part" of $t$. Then, for any $a_{\infty}$ in $\boldsymbol{R}^{\times}$, we get

$$
\begin{aligned}
i_{\infty}\left(\psi_{\infty}\left(a_{\infty} x^{m}\right)\right)= & (2 / m) \Gamma(1 / m)\left(2 \pi\left|a_{\infty}\right|\right)^{-1 / m}-\text { times } \\
& \boldsymbol{e}\left(-\operatorname{sgn}\left(a_{\infty}\right) / 4 m\right) \text { or } \cos (\pi / 2 m)
\end{aligned}
$$

according as $m$ is even or odd. Let $\chi$ denote a character of the group of units of $Z_{p}$ and put

$$
g_{\chi}=p^{-e_{\chi}}\left(\sum_{u \bmod P e_{\chi}} \chi(u) \boldsymbol{e}\left(p^{-e_{\chi}} u\right)\right),
$$

in which $u \neq \equiv 0 \bmod p$ and $e_{x}$ is (as in $\S 1$ ) the smallest positive integer such that $\chi=1$ on $1+p^{e_{x}} \boldsymbol{Z}_{p}$. Then, for any $a_{p}=p^{e_{0}} u_{0}$ in $\boldsymbol{Q}_{p}^{\times}$of order $e_{0}$ satisfying $0 \leqq e_{0}<m$, we get

$$
i_{p}\left(\psi_{p}\left(a_{p} x^{m}\right)\right)=1+(1 / m) \sum_{\alpha^{m}=p}\left(\sum_{\chi^{m=1}} g_{\chi^{-1}} \alpha^{e_{0}+e_{\chi}} \chi\left(u_{0}\right)\right) .
$$

If we take $m=2$, as a special case of what we have recalled, we get $i\left(\psi\left(a x^{2}\right)\right)=1$ for every $a$ in $Q^{\times}$. In particular, we have

$$
i\left(\psi\left(x^{2}\right)\right)=(1+i) \cdot \frac{1}{2}(1-i)=1 .
$$

However, if we take $m=4$ and $a=1$, we get

$$
i\left(\psi\left(x^{4}\right)\right)=(1+\zeta) \cdot \frac{1}{2} \Gamma\left(\frac{1}{4}\right)(2 \pi)^{-(1 / 4)} \zeta^{-1},
$$

in which $\zeta=e(1 / 16)$; this is not even a real number. Finally, if we take $m=3$ and $a=9$, we get $i_{3}\left(\psi_{3}\left(9 x^{3}\right)\right)=0$, hence $i\left(\psi\left(9 x^{3}\right)\right)=0$. (We can show that the situation does not improve even if we extend $\boldsymbol{Q}$ by adjoining $m$-th roots of unity.)

\section{REFERENCES}

[1] P. Deligne, Lectures in honor of W. V. D. Hodge, Cambridge (1973).

[2] B. Dwork, On the zeta function of a hypersurface, Pub. Math. IHES 12 (1962), 5-68; II. Ann. Math. 80 (1964), 227-299.

[ 3 ] H. Hironaka, Resolution of singularities of an algebraic variety over a field of characteristic zero, Ann. Math. 79 (1964), 109-326. 
[4] L. Hörmander, Linear partial differential operators, Grundl. Math. Wiss. 116, Springer-Verlag (1964).

[5] J. Igusa, On the arithmetic of Pfaffians, Nagoya Math. J. 47 (1972), 169-198.

[6] J. Igusa, Complex powers and asymptotic expansions, cited AE (to appear).

[ 7 ] C. L. Siegel, Analytische Zahlentheorie. I, Lect. Note, Göttingen (1963).

[8] A. Weil, Sur certains groupes d'opérateurs unitaires, Acta Math. 111 (1964), 143-211.

[9] A. Weil, Sur la formule de Siegel dans la théorie des groupes classiques, Acta Math. 113 (1965), 1-87.

The Johns Hopkins University 